\title{
Perturbative expansion for master equation and its applications
}

\author{
X. X. Yi ${ }^{a, b}, \mathrm{C} \cdot \mathrm{Li}^{a}$ J. C. $\mathrm{Su}^{c}$ \\ ${ }^{a}$ Institute of Theoretical Physics, Northeast Normal University, \\ Changchun 130024, China \\ ${ }^{b}$ Institute of Theoretical Physics, Academia Sinica, Peking 100080, Chind ${ }^{*}$ \\ c Department of Physics, Jilin University, Changchun 130023, China
}

\begin{abstract}
We construct generally applicable small-loss rate expansions for the density operator of an open system. Successive terms of those expansions yield characteristic loss rates for dissipation processes. Three applications are presented in order to give further insight into the context of those expansions. The first application, of a two-level atom coupling to a bosonic environment, shows the procedure and the advantage of the expansion, whereas the second application that consists of a single mode field in a cavity with linewidth $\kappa$ due to partial transmission through one mirror illustrates a practical use of those expansions in quantum measurements, and the third one, for an atom coupled to modes of a lossy cavity shows the another use of the perturbative expansion.
\end{abstract}

\section{PACS numbers:03.65.-w,05.30.Fk,42.50.Dv}

The study of open quantum systems has recently attracted the attention of physicists from various fields: cosmology[1], condensed matter[2], quantum optics[3-7], particle physics[8], quantum measurement[9,10], and quantum computation[11,12]. The problem can be described generally as interest in the effective dynamics of one subsystem of several interacting subsystems. A formal framework to describe the effective dynamics of such a subsystem is set up in ref.[13], and a short-time perturbative expansion for coherence loss has also been constructed[14]. To some extent(for example, if we are interested in a behavior for finite time), however, time is not as good as the loss rate as a perturbative parameter. Motivated by this and recent experimental developments[15-20] as well as the analysis of models related to them[21-24], we construct generally smallloss rate expansions for dissipation. The results suggest that these are useful in many areas such as high- $\mathrm{Q}$ Cavity QED[15-20], quantum computation[11,12,21-26], quantum measurement[27,28], quantum optics[3-7] etc.

We consider an open quantum system, the total Hamiltonian describing such a system is expressed as

$$
H=H_{0}+H_{e n v}+H_{I}
$$

where $H_{0}$ and $H_{e n v}$ indicate the free Hamiltonian of the system and of the environment, respectively. $H_{I}$ is the interaction Hamiltonian between the system and the environment. It is well known that the form of the master equation depends on the precise kind of the system-environment interaction. In order to derive

${ }^{*}$ Corresponding address 
a master equation for a quite general $H_{I}$, let us suppose that, in the Schrödinger picture, $H_{I}$ can be written as $[3,4]$

$$
H_{I}=\hbar \sum_{m}\left(X_{m}^{+} A_{m}+X_{m}^{-} A_{m}^{\dagger}\right)
$$

where the $X_{m}^{ \pm}$are eigenoperators of the system satisfying

$$
\left[H_{0}, X_{m}^{ \pm}\right]= \pm \hbar \omega_{m} X_{m}^{ \pm}
$$

This form is quite general, since any system operator can be decomposed into eigenoperators of $H_{0}$. As shown in ref.[3,4], we can write the master equation in the following form (in the Schrödinger picture)

$$
\begin{aligned}
\dot{\rho}(t) & =-i\left[H_{0}, \rho\right]+\frac{1}{2} \sum_{m} K_{m}\left(2 X_{m}^{-} \rho X_{m}^{+}-X_{m}^{+} X_{m}^{-} \rho-\rho X_{m}^{+} X_{m}^{-}\right) \\
& +\frac{1}{2} \sum_{m} G_{m}\left(2 X_{m}^{+} \rho X_{m}^{-}-X_{m}^{-} X_{m}^{+} \rho-\rho X_{m}^{-} X_{m}^{+}\right),
\end{aligned}
$$

where

$$
\begin{aligned}
& K_{m}=2 \operatorname{Re}\left[\int_{0}^{\infty} d \tau e^{i \omega_{m} \tau} \operatorname{Tr}_{e n v}\left\{A_{m}(\tau) A_{m}^{\dagger}(0) \rho_{e n v}\right\}\right] \\
& G_{m}=2 \operatorname{Re}\left[\int_{0}^{\infty} d \tau e^{i \omega_{m} \tau} \operatorname{Tr}_{e n v}\left\{A_{m}^{\dagger}(\tau) A_{m}(0) \rho_{e n v}\right\}\right]
\end{aligned}
$$

$\rho(t)=\rho\left(t, K_{m}, G_{m}\right)$ stands for the density operator of the system and $\rho_{\text {env }}$ denotes the density operator of the environment. Notice from eq.(4) that $G_{m}$ should vanish at zero temperature $T=0$, while $K_{m}$ should not if $A_{m}$ are indeed destruction operators of some kind. In case the constant $G_{m}$ and $K_{m}$ are smaller than any one of the internal coupling parameters of the system, the density operator may be expanded in powers of $K_{m}$ and $G_{m}[29]$,

$$
\begin{aligned}
\rho\left(t, K_{m}, G_{m}\right) & =\rho(t, 0,0)+\sum_{m} \frac{\partial \rho}{\partial K_{m}} K_{m}+\sum_{m} \frac{\partial \rho}{\partial G_{m}} G_{m} \\
& +\frac{1}{2} \sum_{m, n} \frac{\partial^{2} \rho}{\partial K_{m} \partial K_{n}} K_{m} K_{n}+\frac{1}{2} \sum_{m, n} \frac{\partial^{2} \rho}{\partial G_{m} \partial G_{n}} G_{m} G_{n} \\
& +\sum_{m, n} \frac{\partial^{2} \rho}{\partial G_{m} \partial K_{n}} G_{m} K_{n}+\ldots
\end{aligned}
$$

Substituting this expression into the master equation, we find the following set of equations

$$
\begin{aligned}
\dot{\rho}(t, 0,0) & =-i\left[H_{0}, \rho(t, 0,0)\right] \\
\frac{\partial \dot{\rho}}{\partial G_{m}} & =-i\left[H_{0}, \frac{\partial \rho}{\partial G_{m}}\right]+\frac{1}{2}\left(2 X_{m}^{+} \rho(t, 0,0) X_{m}^{-}-X_{m}^{-} X_{m}^{+} \rho(t, 0,0)-\rho(t, 0,0) X_{m}^{-} X_{m}^{+}\right) \\
\frac{\partial \dot{\rho}}{\partial K_{m}} & =-i\left[H_{0}, \frac{\partial \rho}{\partial K_{m}}\right]+\frac{1}{2}\left(2 X_{m}^{-} \rho(t, 0,0) X_{m}^{+}-X_{m}^{+} X_{m}^{-} \rho(t, 0,0)-\rho(t, 0,0) X_{m}^{+} X_{m}^{-}\right) \\
\frac{\partial^{2} \dot{\rho}}{\partial K_{m} \partial K_{n}} & =-i\left[H_{0}, \frac{\partial \rho}{\partial K_{m} \partial K_{n}}\right]+\frac{1}{2}\left(2 X_{m}^{-} \frac{\partial \rho}{\partial K_{n}} X_{m}^{+}-X_{m}^{+} X_{m}^{-} \frac{\partial \rho}{\partial K_{n}}-\frac{\partial \rho}{\partial K_{n}} X_{m}^{+} X_{m}^{-}\right) \\
& +\frac{1}{2}\left(2 X_{n}^{-} \frac{\partial \rho}{\partial K_{m}} X_{n}^{+}-X_{n}^{+} X_{n}^{-} \frac{\partial \rho}{\partial K_{m}}-\frac{\partial \rho}{\partial K_{m}} X_{n}^{+} X_{n}^{-}\right)
\end{aligned}
$$




$$
\begin{aligned}
\frac{\partial^{2} \dot{\rho}}{\partial G_{m} \partial G_{n}} & =-i\left[H_{0}, \frac{\partial \rho}{\partial G_{m} \partial G_{n}}\right]+\frac{1}{2}\left(2 X_{m}^{+} \frac{\partial \rho}{\partial G_{n}} X_{m}^{-}-X_{m}^{-} X_{m}^{+} \frac{\partial \rho}{\partial G_{n}}-\frac{\partial \rho}{\partial G_{n}} X_{m}^{-} X_{m}^{+}\right) \\
& +\frac{1}{2}\left(2 X_{n}^{+} \frac{\partial \rho}{\partial G_{m}} X_{n}^{-}-X_{n}^{-} X_{n}^{+} \frac{\partial \rho}{\partial G_{m}}-\frac{\partial \rho}{\partial G_{m}} X_{n}^{-} X_{n}^{+}\right) \\
\frac{\partial^{2} \dot{\rho}}{\partial K_{m} \partial G_{n}} & =-i\left[H_{0}, \frac{\partial \rho}{\partial K_{m} \partial G_{n}}\right]+\frac{1}{2}\left(2 X_{m}^{-} \frac{\partial \rho}{\partial G_{n}} X_{m}^{+}-X_{m}^{+} X_{m}^{-} \frac{\partial \rho}{\partial G_{n}}-\frac{\partial \rho}{\partial G_{n}} X_{m}^{+} X_{m}^{-}\right) \\
& +\frac{1}{2}\left(2 X_{n}^{+} \frac{\partial \rho}{\partial K_{m}} X_{n}^{-}-X_{n}^{-} X_{n}^{+} \frac{\partial \rho}{\partial K_{m}}-\frac{\partial \rho}{\partial K_{m}} X_{n}^{-} X_{n}^{+}\right)
\end{aligned}
$$

Generally speaking, given an initial condition, $\rho(0,0,0)$, we can solve eq.(6) exactly, which gives the zeroth order solution for the density operator $\rho(t, 0,0)$. Substituting the zeroth order solution into eq. $(7), \partial \rho / \partial K_{m}$ or $\partial \rho / \partial G_{m}$ can be calculated. Following this procedure, successive terms of the expansion (5) could be worked out, though the calculation is complicated. Some words of caution are now in order. From the mathematical point of view, the expansion (5) holds if and only if the series converges. This may be satisfied easily in physics for a large number of open systems. For example in a high-Q cavity, the loss rate of the atom-cavity system is small enough to permit us to expand the density operator in powers of the loss rate. At the end of this section, we will present some discussion about this point in detail.

To illustrate the advantage of the expansion (5), we present here a simplest model, which describes a two-level atom coupling to a bose-mode environment. The master equation of such a system is given by[3]

$$
\dot{\rho}=-\frac{1}{2} i \Omega\left[\sigma_{z}, \rho\right]+\frac{1}{2} \gamma\left\{2 \sigma^{-} \rho \sigma^{+}-\rho \sigma^{+} \sigma^{-}-\sigma^{+} \sigma^{-} \rho\right\}
$$

with

$$
\gamma=2 \pi \operatorname{Re}\left[\int_{0}^{\infty} e^{i \omega_{m} \tau} \operatorname{Tr}_{e n v}\left\{b_{m}(\tau) b_{m}^{\dagger}(0) \rho_{e n v}\right\}\right],
$$

where $b_{m}^{\dagger}\left(b_{m}\right)$ stands for the creation(annihilation) operator of the m-th mode of the environment, $\Omega$ is the Rabi frequency, and $\sigma_{z}\left(\sigma^{+}, \sigma^{-}\right)$denote the Pauli matrices. To obtain the form of the master equation given in eq.(9), the environment was assumed to be in its vacuum state. According to eq. $(5),\left\langle\sigma_{z}(t)\right\rangle$ reads:

$$
\left\langle\sigma_{z}(t)\right\rangle=\operatorname{Tr}\left(\rho(t, 0,0) \sigma_{z}\right)+\gamma \operatorname{Tr}\left(\frac{\partial \rho}{\partial \gamma} \sigma_{z}\right)+\frac{1}{2} \gamma^{2} \operatorname{Tr}\left(\frac{\partial^{2} \rho}{\partial \gamma^{2}} \sigma_{z}\right)+\ldots
$$

The first term in eq.(10) is $\left\langle\sigma_{z}(0)\right\rangle$. In order to calculate $\operatorname{Tr}\left(\frac{\partial \rho}{\partial \gamma} \sigma_{z}\right)$, we first evaluate $\operatorname{Tr}\left(\frac{\partial \dot{\rho}}{\partial \gamma} \sigma_{z}\right)$, it is given that

$$
\operatorname{Tr}\left(\frac{\partial \dot{\rho}}{\partial \gamma} \sigma_{z}\right)=-\left\langle\sigma_{z}(0)\right\rangle
$$

Using the same procedure as mentioned, we arrive at

$$
\operatorname{Tr}\left(\frac{\partial^{2} \dot{\rho}}{\partial \gamma^{2}} \sigma_{z}\right)=2\left\langle\sigma_{z}(0)\right\rangle t
$$

The eqs.(11,12) together give

$$
\left\langle\sigma_{z}(t)\right\rangle=\left\langle\sigma_{z}(0)\right\rangle-\gamma t\left\langle\sigma_{z}(0)\right\rangle+\frac{\gamma^{2}}{2 !} t^{2}\left\langle\sigma_{z}(0)\right\rangle+\ldots
$$

Using the algebra of Pauli matrices, we obtain straightforwardly from the master equation that

$$
\left\langle\sigma_{z}(t)\right\rangle=\left\langle\sigma_{z}(0)\right\rangle e^{-\gamma t}
$$


A comparison between eq.(14) and (13) shows that for small $\gamma$, the expansions are a quite good approach for the two level dissipative system, and this result is quite general.

Noticing the eq.(13) is expanded in the product of loss rate and time, we present here the other example to show that these expansions are generally in powers of the loss rate, but not in the product of time and the loss rate. Consider the master equation given in eq. (9) As mentioned above, we calculate $\left\langle\sigma_{z}(t)\right\rangle$ in order to illustrate the advantage of the expansions. The results of $\left\langle\sigma_{z}(t)\right\rangle$ show no difference between the shorttime expansions and the small loss-rate expansions. To show the difference between the two expansions, we calculate $\left\langle\sigma_{x}(t)\right\rangle$. For simplicity, we only present the results up to first order of $\gamma$. It follows from eq.(5) that

$$
\left\langle\sigma_{x}(t)\right\rangle=\operatorname{Tr}\left(\rho(t, 0,0) \sigma_{x}\right)+\gamma \operatorname{Tr}\left(\frac{\partial \rho}{\partial \gamma} \sigma_{x}\right)+\ldots
$$

It is easy to show that (setting $\left.\hbar=1,\left\langle\sigma_{x}(0)\right\rangle=1\right\rangle$ )

$$
\operatorname{Tr}\left(\rho(t, 0,0) \sigma_{x}\right)=\cos (\Omega t) .
$$

In order to compute $\operatorname{Tr}\left(\frac{\partial \rho}{\partial \gamma} \sigma_{x}\right)$, we have to calculate $\operatorname{Tr}\left(\frac{\partial \dot{\rho}}{\partial \gamma} \sigma_{x}\right)$. Based on the expansions, we arrive at

$$
\operatorname{Tr}\left(\frac{\partial \dot{\rho}}{\partial \gamma} \sigma_{x}\right)=\operatorname{Tr}\left(-i\left[H_{0}, \frac{\partial \rho}{\partial \gamma} \sigma_{x}\right]\right)+\frac{1}{2}\left(2 \sigma^{-} \rho(t, 0,0) \sigma^{+} \sigma_{x}-\rho(t, 0,0) \sigma^{+} \sigma^{-} \sigma_{x}-\sigma^{+} \sigma^{-} \rho(t, 0,0) \sigma_{x}\right) \equiv a+b .
$$

Simple calculation gives

$$
a=-\Omega \operatorname{Tr}\left(\frac{\partial \rho}{\partial \gamma} \sigma_{y}\right)
$$

As state above, in order to compute $a$, we have to calculate $\operatorname{Tr}\left(\frac{\partial \dot{\rho}}{\partial \gamma} \sigma_{y}\right)$. Using the same procedure as above, we show that

$$
a=-\Omega^{2} \int_{0}^{t} d t \operatorname{Tr}\left(\frac{\partial \rho}{\partial \gamma} \sigma_{x}\right)-(\cos (\Omega t)-1)
$$

and

$$
b=-\cos (\Omega t) .
$$

The results for $a$ and $b$ together give

$$
\frac{\partial^{2} y(t)}{\partial t^{2}}+\Omega^{2} y(t)=2 \Omega \sin (\Omega t)
$$

with $y(t) \equiv \operatorname{Tr}\left(\frac{\partial \rho}{\partial \gamma} \sigma_{x}\right)$ and initial conditions $y(t=0)=0,\left.\dot{y}(t)\right|_{t=0}=-1$. This is a two order differential equation and can be solved easily, once $y(t)$ is known, $\left\langle\sigma_{x}(t)\right\rangle$ up to first order of $\gamma$ is given. It is obvious that results given above are indeed different from the short time expansions, since the results given by short time expansions are in powers of time $t$.

The results up to first order of $\gamma(15)$ and an exact numerical results are illustrated in Fig.1. The parameters chosen are $\Omega=2$, and time is in units of $\Omega$. In Fig.1 the scattering line represents the exact numerical results, whereas the dot line and the solid line show the results from the expansion. The dot line and the solid line are for different $\gamma$, and $\gamma$ for dot line is smaller than one in solid line, those curves show that the expansions (15) are indeed a good approximation to the exact solution. 
We need to point out that for most open systems, average values of meaningful quantities can't be obtained exactly in any way. Therefore the expansions of the density operator provide a practical approach to the exact solution. For example, consider a single-mode field in a lossy cavity. The density operator for that mode obeys the following master equation in the Schrödinger picture[3,4],

$$
\dot{\rho}=-i\left[\omega_{f} a^{\dagger} a, \rho\right]+\frac{\kappa}{2}\left(2 a \rho a^{\dagger}-a^{\dagger} a \rho-\rho a^{\dagger} a\right)
$$

where $\kappa$ is the linewidth of the cavity mode with frequency $\omega_{f}$. In most textbooks, the solution of the master equation is given in terms of diagonal matrix elements $\langle n|\rho| n\rangle$ in a stationary state. Given an initial condition for the density operator, the evolution of $\rho$, however, is more useful than the stationary solution. In what follows, we present a solution of the master equation in a number state (Fock state) basis.

For a high-Q cavity, the linewidth $\kappa$ due to partial transmission through one mirror is so small that we can expand $\rho$ in powers of $\kappa$ :

$$
\rho(t)=\rho^{0}(t)+\frac{\partial \rho}{\partial \kappa} \kappa+\frac{1}{2} \frac{\partial^{2} \rho}{\partial \kappa^{2}} \kappa^{2}+\ldots,
$$

In a number state basis $\{|n\rangle, n=0,1,2,3 \ldots\}$, the expansion can be written as

$$
\rho(t)=\sum_{m, n} \rho_{m n}^{0}(t)|m\rangle\left\langle n\left|+\sum_{m, n} \sum_{k=1}^{\infty} \frac{1}{k !} \frac{\partial^{k} \rho_{m n}}{\partial \kappa^{k}}\right| m\right\rangle\langle n| \kappa^{k} .
$$

Here the subscripts on the density operator $\rho_{m n}$ indicate matrix elements of $\rho$ in the number basis and $\rho_{0}$ is the solution of eq.(16) with $\kappa=0$. With this notation, it follows from eqs. $(6),(7)$ and (8) that

$$
\begin{aligned}
& \rho_{m n}^{0}(t)=\rho_{m n}^{0}(0) e^{-i(m-n) \omega_{f} t} \\
& \frac{\partial^{k} \rho_{m n}}{\partial \kappa^{k}}=c_{k}(t) e^{-i \omega_{f}(n-m) t}
\end{aligned}
$$

This iterative equation gives the density operator expansions of the system under consideration. Here, $\rho_{m n}(0)$ stands for the initial condition of $\rho$, and

$$
\begin{aligned}
& c_{k}(t)=\int_{0}^{t} F_{k}\left(t^{\prime}\right) e^{i \omega_{f}(n-m) t^{\prime}} d t^{\prime} \\
& F_{1}(t)=\sqrt{(n+1)(m+1)} \rho_{m+1, n+1}^{0}(t)-\frac{1}{2} m \rho_{m n}^{0}(t)-\frac{1}{2} n \rho_{m n}^{0}(t), \\
& F_{k}(t)=\sqrt{(n+1)(m+1)} \frac{\partial^{k-1} \rho_{m+1, n+1}}{\partial \kappa^{k-1}}-\frac{m+n}{2} \frac{\partial^{k-1} \rho_{m, n}}{\partial \kappa^{k-1}}, k=2,3,4 \ldots
\end{aligned}
$$

The master equation in the form (16) is widely used in field-quadrature measurement[27,28]. As shown in Ref.[27,28], different approximations to eq.(16) correspond to different measurement schemes, therefore the expansions(6-8) for the density operator provide a new method to develop quantum measurement theory. In contrast to the short time perturbative expansions[14], the expansions (6-8) hold for finite time as long as the linewidth $\kappa$ is small. In other words, whether the expansions hold does not depend on time $t$.

In addition to quantum measurement, these expansions have use in high-Q cavity QED. There are many interesting features in cavity QED. One of them is spontaneous emission. Spontaneous emission is so fundamental that it is usually regarded as an inherent property of matter. The master equation for a single atom coupling to a mode of a lossy cavity is given in the interaction picture under rotating-wave and dipole approximations by[15] 


$$
\dot{\rho_{I}}=\frac{\gamma}{2}\left(2 \sigma^{-} \rho_{I} \sigma^{+}-\sigma^{+} \sigma^{-} \rho_{I}-\rho_{I} \sigma^{+} \sigma^{-}\right)+\frac{\kappa}{2}\left(2 a \rho_{I} a^{\dagger}-a^{\dagger} a \rho_{I}-\rho_{I} a^{\dagger} a\right),
$$

where $\rho_{I}$ stands for the reduced density operator of the system that consists of an atom and a cavity mode, $\gamma$ denotes the linewidth of the atom, and $\kappa$ describes the loss rate of the cavity.This is different from eq.(9) in which the loss of the single-mode field is neglected. It is well known that the emission spectrum may be expressed in terms of average values of the atom operator $\vec{\sigma}$. In this sense, we may calculate the average value of the atom operator to replace computing the emission spectrum without any loss of generality. Moreover, the study of many other effects in cavity QED such as atomic dipole squeezing[30], population trapping[31], and atomic collapse-and-revival phenomenon[32] may be reduced to calculate and analyse the average value of the atom operator. In the remainder of this paper, based on the expansion scheme, we compute the average value of an atom operator given by $A=\lambda^{(+)} \sigma^{+}+\lambda^{(-)} \sigma^{-}+\lambda^{(z)} \sigma_{z}$. For this end, we first of all list the expansions of the density operator $\rho_{I}(t)$ in the interaction picture

$$
\rho_{I}(t)=\rho_{I}^{0}(t)+\frac{\partial \rho_{I}(t)}{\partial \kappa} \kappa+\frac{\partial \rho_{I}(t)}{\partial \gamma} \gamma+\frac{1}{2} \frac{\partial^{2} \rho_{I}(t)}{\partial \kappa^{2}} \kappa^{2}+\frac{1}{2} \frac{\partial^{2} \rho_{I}(t)}{\partial \gamma^{2}} \gamma^{2}+\frac{1}{2} \frac{\partial^{2} \rho_{I}(t)}{\partial \gamma \partial \kappa} \gamma \kappa+\ldots
$$

Here,

$$
\begin{gathered}
\dot{\rho}_{I}^{0}(t)=0 \\
\frac{\partial \dot{\rho}_{I}(t)}{\partial \gamma}=\frac{1}{2}\left(2 \sigma^{-} \rho_{I}^{0}(t) \sigma^{+}-\sigma^{+} \sigma^{-} \rho_{I}^{0}(t)-\rho_{I}^{0}(t) \sigma^{+} \sigma^{-}\right), \\
\frac{\partial \dot{\rho}_{I}(t)}{\partial \kappa}=\frac{1}{2}\left(2 a \rho_{I}^{0}(t) a^{\dagger}-a^{\dagger} a \rho_{I}^{0}(t)-\rho_{I}^{0}(t) a^{\dagger} a\right), \\
\frac{\partial^{2} \dot{\rho}_{I}(t)}{\partial \gamma^{2}}=\frac{1}{2}\left(2 \sigma^{-} \frac{\partial \rho_{I}}{\partial \gamma} \sigma^{+}-\sigma^{+} \sigma^{-} \frac{\partial \rho_{I}}{\partial \gamma}-\frac{\partial \rho_{I}}{\partial \gamma} \sigma^{+} \sigma^{-}\right), \\
\frac{\partial^{2} \dot{\rho}_{I}(t)}{\partial \kappa^{2}}=\frac{1}{2}\left(2 a \frac{\partial \rho_{I}}{\partial \kappa} a^{\dagger}-a^{\dagger} a \frac{\partial \rho_{I}}{\partial \kappa}-\frac{\partial \rho_{I}}{\partial \kappa} a^{\dagger} a\right), \\
\frac{\partial \rho_{I}(t)}{\partial \gamma \partial \kappa}=\frac{\partial \rho_{I}}{2}\left(2 \sigma^{+}-\sigma^{+} \sigma^{-} \frac{\partial \rho_{I}}{\partial \kappa}-\frac{\partial \rho_{I}}{\partial \kappa} \sigma^{+} \sigma^{-}\right)+\frac{1}{2}\left(2 a \frac{\partial \rho_{I}}{\partial \gamma} a^{\dagger}-a^{\dagger} a \frac{\partial \rho_{I}}{\partial \gamma}-\frac{\partial \rho_{I}}{\partial \gamma} a^{\dagger} a\right)
\end{gathered}
$$

It is easy to show that for any atom operator $A$,

$$
\operatorname{Tr}\left(\frac{\partial^{n} \dot{\rho}_{I}(t)}{\partial \kappa^{n}} A\right)=\operatorname{Tr}\left(\frac{\partial^{n} \dot{\rho}_{I}(t)}{\partial \kappa^{m} \partial \gamma^{n-m}} A\right)=0
$$

for $n \geq m \neq 0$, while

$$
\begin{aligned}
\operatorname{Tr}\left(\frac{\partial \dot{\rho}_{I}}{\partial \gamma} A\right) & =\frac{1}{2} \operatorname{Tr}\left(\rho^{0}(t) B\right) \\
\operatorname{Tr}\left(\frac{\partial^{n} \dot{\rho}_{I}}{\partial \gamma^{n}} A\right) & =\frac{1}{2} \operatorname{Tr}\left(\frac{\partial^{n-1} \rho_{I}}{\partial \gamma^{n-1}} B\right)
\end{aligned}
$$

where $B=-4 \lambda^{(+)} \sigma^{+}-4 \lambda^{(-)} \sigma^{-}$. Eqs.(23) and (24) suggest that the average value for any atom operator can be calculated analytically as an expansion in powers of $\kappa$ and $\gamma$, provided $\rho^{0}(t)$ (the zeroth order density 
operator in the Schrödinger picture) is known. Generally speaking, given a initial condition for $\rho$, the $\rho^{0}(t)$ that obeys the von Neumann equation can be given readily. In the model presented above, the von Neumann equation is given by

$$
\dot{\rho}^{0}=-i\left[H_{0}, \rho^{0}\right],
$$

Where $H_{0}$ denotes the free Hamiltonian for the cavity-atom system (Jaynes-Cummings model)

$$
H_{0}=\omega_{f} a^{\dagger} a+\frac{1}{2} \omega_{a} \sigma_{z}+g\left(a^{\dagger} \sigma^{-}+\sigma^{+} a\right)
$$

If the cavity-atom system is initially in a state $|e, n\rangle=|e\rangle \otimes|n\rangle$, i.e. the atom is in its excited state, while the single-mode cavity is in the number state $|n\rangle$, then $\rho^{0}(t)$ reads

$$
\rho^{0}(t)=\left|\psi^{0}(t)\right\rangle\left\langle\psi^{0}(t)\right|,
$$

where

$$
\begin{gathered}
\left|\psi^{0}(t)\right\rangle=\frac{1}{2} \sin \theta_{n+1}\left(e^{-i E_{+}(n+1) t}-e^{-i E_{-}(n+1) t}\right)|g, n+1\rangle \\
+\left(\sin ^{2} \frac{\theta_{n+1}}{2} e^{-i E_{+}(n+1) t}+\cos ^{2} \frac{\theta_{n+1}}{2} e^{-i E_{-}(n+1) t}\right)|e, n\rangle . \\
E_{ \pm}(n+1)=\frac{\omega_{f}}{2}(2 n+1) \pm \sqrt{\delta^{2}+g^{2}(n+1)}, \theta_{n+1}=\operatorname{arctg} \frac{2 g \sqrt{n+1}}{\delta}, \delta=\omega_{f}-\omega_{a} .
\end{gathered}
$$

It follows from eq. $(6,7,8)$ that

$$
\begin{aligned}
\operatorname{Tr}\left(\rho^{0}(t) A\right)= & \frac{1}{4} \operatorname{Tr}\left(\rho_{0}(t) B\right)+\lambda^{(z)} \sum_{n}\left\{\left|\sin ^{2} \frac{\theta_{n+1}}{2} e^{-i E_{+}(n+1) t}+\cos ^{2} \frac{\theta_{n+1}}{2} e^{-i E_{-}(n+1) t}\right|^{2}\right. \\
- & \left.\sin ^{2} \theta_{n+1} \sin ^{2}\left(\sqrt{\delta^{2}+g^{2}(n+1)} t\right)\right\} \\
\operatorname{Tr}\left(B \rho^{0}(t)\right)= & \sum_{n}\left\{-2 \lambda^{(+)} \sin \theta_{n}\left(e^{-i E_{+}(n) t}-e^{-i E_{-}(n) t}\right)\right. \\
& \left(\sin ^{2} \frac{\theta_{n+1}}{2} e^{i E_{+}(n+1) t}+\cos ^{2} \frac{\theta_{n+1}}{2} e^{i E_{-}(n+1) t}\right)- \\
& 2 \lambda^{(-)}\left(\sin ^{2} \frac{\theta_{n+1}}{2} e^{-i E_{+}(n+1) t}+\cos ^{2} \frac{\theta_{n+1}}{2} e^{-i E_{-}(n+1) t}\right) \\
& \left.\sin \theta_{n}\left(e^{i E_{+}(n) t}-e^{i E_{-}(n) t}\right)\right\} .
\end{aligned}
$$

Then successive perturbative terms of average value up to second order of $\gamma$ for an atom operator are given by

$$
\langle A\rangle(t)=\operatorname{Tr}\left(\rho_{0}(t) A\right)+\frac{\gamma}{2} \int_{0}^{t} \operatorname{Tr}\left(\rho^{0}\left(t^{\prime}\right) B\right) d t^{\prime}-\frac{\gamma^{2}}{2} \int_{0}^{t} d t^{\prime} \int_{0}^{t^{\prime}} \operatorname{Tr}\left(\rho^{0}\left(t^{\prime \prime}\right) B\right) d t^{\prime \prime}+\ldots
$$

Based on the short-time expansion, $\langle A\rangle(t)=\operatorname{Tr}(A \rho(t)) \simeq A_{0}+A_{1} t+A_{2} t^{2}+\ldots$, in powers of $t$, where $A_{0}=\operatorname{Tr}(A \rho(0)), A_{1}=\operatorname{Tr}\left(A \frac{\partial \rho}{\partial t}(0)\right)$ and $A_{2}=\frac{1}{2} \operatorname{Tr}\left(A \frac{\partial^{2} \rho}{\partial t^{2}}(0)\right)$. This is quite different from the results given by eq.(31).

Although we are currently investigating the perturbative expansion for an open system, we opt here for a few qualitative comments. Mathematically, the perturbative expansion is a good approach to the exact 
solution of the master equation so long as loss rates $\gamma$ and $\kappa$ are smaller than all other internal coupling constants of the system. This condition holds for high-Q cavities from the physical point of view. In fact, an optical cavity of $\sim 20 \mu \mathrm{m}$ diameter has $\mathrm{g} / 2 \pi \sim 125 \mathrm{MHz}$ and $\kappa / 2 \pi \sim 100 \mathrm{KHz}$ for reasonable $Q \sim 10^{9}$. Thus the ratio $g / \kappa \sim 10^{3}$. Even $g / \kappa \sim 10^{4}$ seems feasible for microspheres[33]. Generally, in the optical domain $g / \gamma \sim 10^{2}$, great enough for the perturbative expansion in powers of $\gamma$ to hold.

In the end of this paper, we turn our attention to study the decoherence in $N$ two-level atoms. This problem is usually related to the register in quantum computer. A few papers[11,12,34] have been published on this subject, but a key additional feature of the present paper is to study the decoherence from a new aspect. If the system consists of $N$ two-level atoms, the decoherence is due to the inevitable coupling of the $N$ atoms to the external environment. Generally, the environment may be treated as that consists of an infinite number of oscillators. The Hamiltonian describing such decoherence process takes the form

$$
\begin{aligned}
H & =H_{s}+H_{e n v}+H_{I}, \\
H_{s} & =\sum_{i=1}^{N} \Omega_{i} \sigma_{i}^{z}, \\
H_{e n v} & =\sum_{k=1}^{\infty} \omega_{k} b_{k}^{\dagger} b_{k}, \\
H_{I} & =\sum_{i=1}^{N} \sum_{k=1}^{\infty}\left(g_{k i} b_{k}^{\dagger} \sigma_{i}^{-}+\text {h.c. }\right),
\end{aligned}
$$

where $\sigma_{i}^{\alpha}$ are the spin- $\frac{1}{2}$ Pauli operators ( $i$ denotes the qubit index) and $b_{k}, b_{k}^{\dagger}$ are the bosonic operators, $H_{s}, H_{e n v}$ are the free Hamiltonian of the system and the environment, respectively. And $H_{I}$ stands for the $N$ qubits-environment interaction. This model is closely related to the Dicke maser model[35,36]. The Hamiltonian (32) is complicated so it is hard to find its exact solution though the Hilbert space associated with this model can split into invariant eigenspaces[34]. Fortunately, with the perturbative approach created in the previous section, the complex system can be easily treated. To start with, we give the master equation of the system

$$
\begin{aligned}
\dot{\rho} & =-i[H, \rho]+\frac{1}{2}\left\{\sum_{i} K_{i}\left(2 \sigma_{i}^{-} \rho \sigma_{i}^{+}-\rho \sigma_{i}^{+} \sigma_{i}^{-}-\sigma_{i}^{+} \sigma_{i}^{-} \rho\right)\right\} \\
& +\frac{1}{2}\left\{\sum_{i} G_{i}\left(2 \sigma_{i}^{+} \rho \sigma_{i}^{-}-\sigma_{i}^{-} \sigma_{i}^{+} \rho-\rho \sigma_{i}^{-} \sigma_{i}^{+}\right)\right\} \\
& =-i[H, \rho]+\mathcal{L} \rho .
\end{aligned}
$$

Here

$$
\begin{gathered}
K_{m}=2 \operatorname{Re} \int d \tau e^{i \Omega_{m} \tau} \operatorname{Tr}_{e n v}\left\{A_{m}(\tau) A_{m}^{\dagger}(0) \rho_{e n v}\right\} \\
G_{m}=2 \operatorname{Re} \int d \tau e^{i \Omega_{m} \tau} \operatorname{Tr}_{e n v}\left\{A_{m}^{\dagger}(\tau) A_{m}(0) \rho_{e n v}\right\} \\
A_{m}(\tau)=\sum_{j=1}^{\infty} \hbar g_{m j} b_{j} e^{-i \omega_{j} \tau} .
\end{gathered}
$$


In the case of so-called Dicke limit $[35,36], A_{m}$ does not depend on the atom index $m$. This holds, for example, when the typical environment wavelengths are much greater than the distances between the atoms.

In order to study the decoherence of the atoms, we assume that the initial state of the system is

$$
\left.\left|\psi_{m}\right\rangle=S_{m}^{+}|0\rangle+\prod_{j=m+1}^{N}|0\rangle\right)
$$

where $S_{m}^{+}=\prod_{j=1}^{m \leq N} \sigma_{j}^{+}|0\rangle$, and $|0\rangle=|0\rangle_{1} \otimes|0\rangle_{2} \otimes \ldots \otimes|0\rangle_{N}$ stands for the lower state of the atoms. Eq.(34) indicates that there are $m$ atoms in the upper state $|1\rangle$, and the rest of the $N$ atoms are in their lower state. With those initial conditions, the probability of the atoms remaining in the initial state is given by $F_{m}(t)$

$$
F_{m}(t)=1-\frac{K}{\Gamma_{K 1}}-\frac{K^{2}}{\Gamma_{K 2}}-\frac{G}{\Gamma_{G 1}}-\frac{G^{2}}{\Gamma_{G 2}}-\frac{G K}{\Gamma_{G K}}+O\left(G^{3}\right)+O\left(K^{3}\right),
$$

with

$$
\begin{gathered}
\frac{1}{\Gamma_{K 1}}=t[2+2(2 m-N)], \\
\frac{1}{\Gamma_{K 2}}=-t^{2}[2+2(2 m-N)], \\
\frac{1}{\Gamma_{G 1}}=t[2-2(2 m-N)], \\
\frac{1}{\Gamma_{G 2}}=-t^{2}[2-2(2 m-N)], \\
\frac{1}{\Gamma_{G K}}=\frac{1}{2} G K t^{2}[4(2 m-N)-4] .
\end{gathered}
$$

Here, we suppose that all qubits are alike, so $\Omega_{m}=\Omega$ and $K_{m}=K$ and $G_{m}=G$. $G$ and $K=G+1$ depend on environment temperature $T$ through $G=1 /[\exp (\hbar \Omega / k T)-1]$, which indicate that the probability decrease with the temperature increasing. In fact, the fidelity in the field of quantum information is nothing but an overlap between the initial and final state of the qubits(two-level system). Eq. (35) suggests that the fidelity depends on $m$ i.e. the number of the atoms in upper state initially. And to get the maximum of the fidelity, the variable $m$ should be taken as small as possible.

To sum up, in this paper, we construct the small-loss rate perturbative expansion for the density operator of an open system. The expansions provide a quite good approach to the exact solution in case the master equation of the system can not be solved exactly. As an interesting application of this expansions, we used it to calculate some average values such as $\sigma_{z}$ and $\sigma_{x}$ in dissipative two-level system, the expansions of the density operator for a single-mode field in a lossy cavity are also presented, and the dynamical property in $N$ two-level atom system.

In addition, the other meaningful quantities of the open system such as energy, occupation probability etc. can be expanded in the same spirit of the density operator, so long as the master equation of the system is known. 
[1] M.Gell-mann, J.B.Hartle. Phys. Rev. D 47(1993)3345.

[2] A.O.Caldeira and A.J.Leggett, Ann. Phys.(N.Y.)149(1983)374.

[3] C.W.Gardiner, Quantum noise (Springer-Verlag),New York,1991.

[4] William H.Louisell, Quantum statistical properties of radiation, John Wiley, 1973.

[5] Xue-Xi Yi,Physica scripta,52(1995)508.

[6] Xue-Xi Yi, J.Mod. Opt. 43(1996)675.

[7] Xue-Xi Yi, J.C.Su J. Phys. B 30(1997)L411. And references therein.

[8] H.T.Elze, Nucl. Phys. B 436(1995)213.

[9] W.H.Zurek, Phys. Rev. D 26(1982)1862.

[10] D.F.Walls, M.J.Collet, G.J.Milburn, Phys. Rev. D 32(1985)3208.

[11] W.G.Unruh, Phys. Rev. A 51(1995)992.

[12] R. Landauer, Philos. Trans. R. Soc. London Ser. A 353(1995)367.

[13] M.C. Nemes and A.F.R.de Toledo Piza, Physica 137A(1986)367.

[14] Ji Il Kim, M.C. Nemes, A.F.R. de Toledo Piza, H.E. Borges, Phys. Rev. Lett. 77(1996)207.

[15] W.Ren and H.J.Carmichael Phys. Rev. A 51(1995)752.

[16] S.Haroche and D.Kleppner, Physics today 42(1989)24.

[17] P.Meystre, Phys. Rep. 219(1992)243.

[18] O.Benson, M.Weidinger, G.Raithel, and H.Walther, J.Mod. Opt. 44(1997)2011.

[19] M.S.Kim, G.Antesberger, C.T.Bodendorf, and H.Walther, Phys. Rev. A 58(1998) R65.

[20] D.W.Vernooy, A.Furusawa, N.Ph.Georgiades,V.S.Ilchenko, H.J.Kimble, Phys. Rev. A 57(1998)R2293.

[21] J.I.Cirac, P.Zoller, Phys. Rev. Lett. 74(1995) 4049.

[22] S.J.Van Enk, J.I.Cirac, and P.Zoller, Phys. Rev. Lett. 78(1997)4293.

[23] J.I.Cirac, P.Zoller, H.J.Kimble, and H.Mabuchi, Phys. Rev.Lett. 78(1997)3221.

[24] S.J.Van Enk, J.I.Cirac, P.Zoller Phys. Rev. Lett. 79(1997)5178.

[25] A special issue on coherence and decoherence, Proc. R. Soc Lond. A 454(1998)257(begin page).

[26] C.H.Bennett, Physics Today, 48(1995)24.

[27] V.B. Braginsky, F. Y. Khalili, Quantum measurement (Cambridge, 1992).

[28] H.M.Wiseman, G.J.Milburn, Phys. Rev. A 47(1993)642.

[29] N.G.van Kmpen, Phys. Rep. 124(1985)69.

[30] T.Nasreen, J. Opt. Soc. Am. B11(1994)386.

[31] B.D.Agap'ev, M.B.Gornyi, and B.G.Matisov, Yu.V.Rozhdestvenskii, Phys. Uspekhi 36(1993)763.

[32] P.R.P.Jushi, S.V.Lawande, Phys. Rev. A 44(1991)2135.

[33] M.L.Gorodetsdy, A.A.Savchenkov, and B.S.Ilchenke, Opt. Lett. 21(1995)453.

[34] P.Zanardi and M.Rasetti, Phys. Rev. Lett. 79(1997)3306.

[35] R.Davidson, J.J.Kozak, J.Math. Phys. 8(1971)903.

[36] K.Hepp, E.Lieb, Phys. Rev. A8(1973)2517.

\section{Figure captions}

Fig.1: $\sigma_{x}(t)$ vs time $t$, the parameter chosen is $\Omega=2$. The scattering line represent the exact numerical results, whereas the solid line and the dot line show the results from the expansion, $\gamma$ in the solid line and the dot line is different, dot line: $\gamma=0.01$, solid line: $\gamma=0.05$. 